\title{
HYDROSTATIC PRESSURE SPECTROSCOPY OF THE VANADIUM LUMINESCENCE IN GaAs
}

\author{
A. Wysmolek, R. BożeK, A. Babiński and A.M. Hennet \\ Institute of Experimental Physics, Warsaw University \\ Hoża 69, 00-681 Warszawa, Poland
}

\begin{abstract}
We report luminescence measurements of the intracenter transition ${ }^{3} T_{2} \rightarrow{ }^{3} A_{2}$ of the $\mathrm{V}^{3+}\left(3 d^{2}\right)$ charge state in semi-insulating $\mathrm{GaAs}$ under hydrostatic pressure up to $0.8 \mathrm{GPa}$ at liquid helium temperature. The hydrostatic pressure coefficient of the zero-phonon line is found to be equal to $6.9 \pm 0.2 \mathrm{meV} / \mathrm{GPa}$. This result enables us to determine the Huang-Rhys parameter, which characterizes the coupling to the symmetric mode of vibration, as $S_{A}=1.4 \pm 0.1$. Using this parameter, computer simulation leading to a reconstruction of the shape of both luminescence and corresponding absorption spectra were performed.
\end{abstract}

PACS numbers: 71.55.Eq, 78.55.Cr

\section{Introduction}

Optical absorption and luminescence bands in GaAs at $0.74 \mathrm{eV}$ corresponding to ${ }^{3} A_{2} \leftrightarrow{ }^{3} T_{2}$ transitions of the $\mathrm{V}^{3+}\left(3 d^{2}\right)$ charge state crystals have been investigated for many years (see for example review articles $[1,2]$ ). The results of several experiments were analysed in terms of a dynamical Jahn-Teller effect with the $\varepsilon$-mode existing in the excited ${ }^{3} T_{2}$ state [3-7]. The obtained values of the Huang-Rhys parameter $S_{E}=E_{\mathrm{JT}} / \hbar \omega_{E}$ (where $E_{\mathrm{JT}}$ is the Jahn-Teller energy and $\hbar \omega_{E}$ - the phonon energy) vary between 3.4 and 4 . It was also shown [8] that in order to explain the total shape of luminescence and absorption bands, an interaction with the symmetric $\alpha$-mode of vibrations should also be taken into account.

The lack of any effect of hydrostatic pressure up to $1 \mathrm{GPa}$ on the transport properties of $p$-type GaAs:V [9] suggests a minor influence of the $\alpha$-mode on the ground ${ }^{3} A_{2}$ state (degenerate with the GaAs valence band). The parameter $S_{A}$ describing the interaction of the $\alpha$-mode with the excited ${ }^{3} T_{2}$ state can be thus obtained by a moment analysis of the luminescence or absorption bands [8] or from the hydrostatic pressure coefficient of one of these bands. This pressure coefficient is known from a uniaxial stress experiment [3], but with a significant experimental error. 
This paper presents hydrostatic pressure measurements of the vanadium luminescence band which gave a much more accurate value of the pressure coefficient and, subsequently the $S_{A}$ parameter. This result and the $\varepsilon$-mode Jahn-Teller data from literature make it possible to reproduce the shape of both luminescence and absorption spectra

\section{Experimental}

Vanadium doped, semi-insulating GaAs crystals were prepared by the liquid encapsulated Czochralski (LEC) technique with a vanadium concentration of $7 \div 8 \times 10^{16} \mathrm{~cm}^{-3}$ [10]. Zero pressure luminescence was measured in a CF1204 (Oxford) cryostat. For pressure investigations samples were placed in a high pressure optical cell with benzine as the transmitting medium. The cell with a sample was mounted in the exchange helium gas cryostat and cooled to low temperatures. $\mathrm{Hy}$ drostatic pressure up to $0.8 \mathrm{GPa}$ was measured with a calibrated InSb manometer. Photoluminescence (PL) was excited by the $488 \mathrm{~nm}$ line of an $\mathrm{Ar}$ ion laser with power density around $1 \mathrm{~W} / \mathrm{cm}^{2}$. PL was analysed with a SPEX 500M monochromator equipped with a North Coast Optics Ge photodiode or cooled $\mathrm{PbS}$ detector (Hamamatsu).

\section{Results and discussion}

A typical low-temperature $(4 \mathrm{~K})$ atmospheric pressure photoluminescence spectrum related to the ${ }^{3} T_{2} \rightarrow{ }^{3} A_{2}$ transition is shown in Fig. 1 . It consists of a strong zero-phonon line (ZPL) A with accompanying hot line $\mathrm{B}$ (see inset)
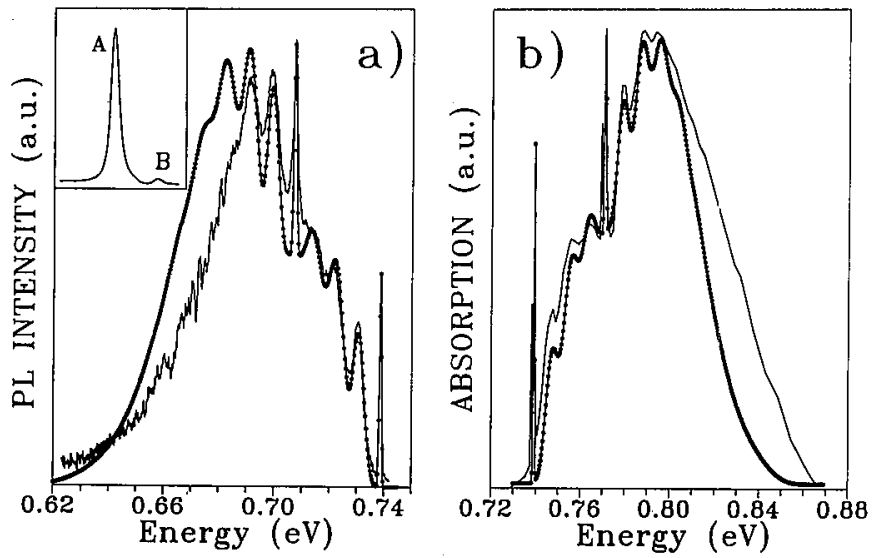

Fig. 1. Luminescence (a) and absorption (b) bands corresponding to ${ }^{3} A_{2} \leftrightarrow{ }^{3} T_{2}$ transitions of the $\mathrm{V}^{3+}\left(3 d^{2}\right)$ charge state in GaAs at about $4 \mathrm{~K}$ (solid lines). Theoretical Poisson shapes (star lines) were calculated for the parameters $S_{E}=3.9, \hbar \omega_{E}=8.1 \mathrm{meV}$; $S_{A}=1.4, \hbar \omega_{E}=31.5 \mathrm{meV}$. Inset - luminescence zero-phonon lines A and B. 


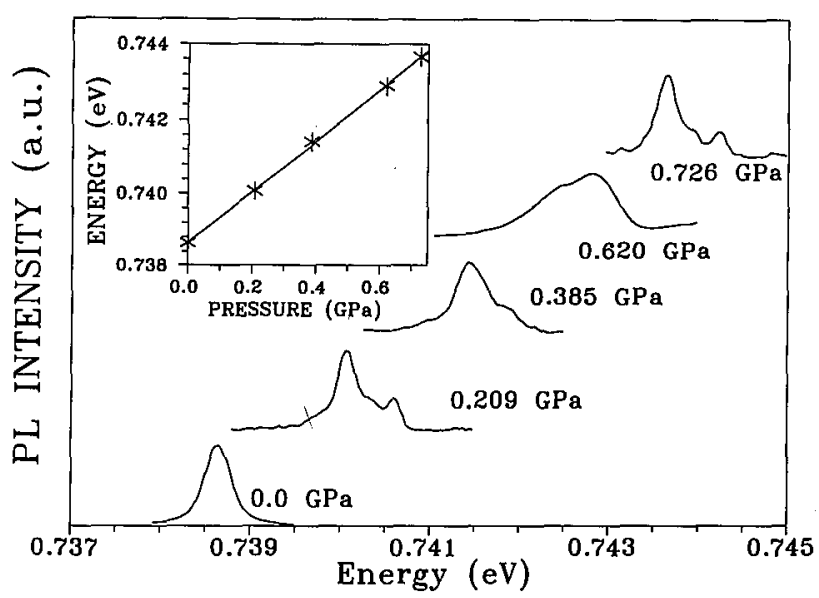

Fig. 2. Shift of the luminescence zero-phonon line A under hydrostatic pressure at about $4 \mathrm{~K}$ (10 $\mathrm{K}$ for $0.62 \mathrm{GPa}$ ). Inset - a linear fit to the experimental data with a pressure coefficient equal to $6.9 \pm 0.2 \mathrm{meV} / \mathrm{GPa}$.

followed by phonon replicas. The energy shift of the $\mathrm{A}$ line with increasing pressure is presented in Fig. 2. With the exception of the measurements at $0.62 \mathrm{GPa}$, all photoluminescence data were collected at about $4 \mathrm{~K}$. The low intensity shoulders of the main line are due to axial stresses induced on the GaAs surface by frozen benzene. The measured positions of line $A$ were plotted versus the stress value. A straight line fit to the data gave the hydrostatic pressure coefficient of the ZPL being equal to $6.9 \pm 0.2 \mathrm{meV} / \mathrm{GPa}$. From this value it is easy to calculate the symmetric vibration energy $E_{A}=43 \pm 3 \mathrm{meV}$. Taking the phonon energy $\hbar \omega_{A}=31.5 \mathrm{meV}$ from the experimental spectra one obtains the Huang-Rhys parameter being equal to $S_{A}=E_{A} / \hbar \omega_{A}=1.4 \pm 0.1$.

Computer simulation taking into account optical transitions involving two phonons ( $\hbar \omega_{E}$ and $\left.\hbar \omega_{A}\right)$ with the $\varepsilon$-mode Jahn-Teller effect at the ${ }^{3} T_{2}$ state known from other experiments [3-7] and the obtained value of the $S_{A}$ parameter lead to a reconstruction of the shape of both luminescence and absorption bands (see Fig. 1).

\section{Acknowledgment}

This work was supported by the Committee for Scientific Research grant No. 201799101.

\section{References}

[1] B. Clerjaud, J. Phys. C Solid State Phys. 18, 3615 (1985).

[2] A.M. Hennel, in: Imperfections in III-V Materials, Ed. E. Weber; in series Semiconductors and Semimetals, Eds. R.K. Willardson, A.C. Beer, Vol. 38, Academic Press, in press. 
[3] G. Armelles, J. Barrau, D. Thébaut, J. Phys. C, Solid State Phys. 17, 6883 (1984).

[4] G. Armelles, J. Barrau, D. Thébaut, M. Brousseau, J. de Phys. 45, 1795 (1984).

[5] G. Aszodi, U. Kaufmann, Phys. Rev. B 32, 7108 (1985).

[6] K.J. Nash, M.S. Skolnik, B. Cockayne, W.R. MacEwan, J. Phys. C, Solid State Phys. 17, 6199 (1984).

[7] F.G. Anderson, F.S. Ham, in: Defects in Semiconductors, Ed. G. Ferenczi, Material Science Forum 38-41, 305 (1989).

[8] A. Wysmolek, Z. Liro, A.M. Hennel, in: Defects in Semiconductors, Ed.G. Ferenczi, Material Science Forum 38-41, 827 (1989).

[9] D. Wasik, A.M. Hennel, unpublished.

[10] A.M. Hennel, C.D. Brandt, K.Y. Ko, J. Lagowski, H.C. Gatos, J. Appl. Phys. 62, 163 (1987). 